\title{
Multiple vulvar dilated pores in a postmenopausal woman*
}

Ge Shi ${ }^{1}$

Ni Liang ${ }^{1}$

Ying Zhou ${ }^{1}$

\author{
Wei $\mathrm{Li}^{1}$ \\ Ting-Ting Wang ${ }^{1}$ \\ Yi-Ming Fan $^{1}$
}

DOI: http:/ / dx.doi.org/10.1590/abd1806-4841.20175434

\begin{abstract}
Winer's dilated pore is an infrequent appendageal tumor characterized by a giant comedone on the face, neck, and upper trunk in adults. We report a 57-year-old woman who developed multiple asymptomatic black papules on both labia majora. Histopathology showed grouped dilated follicles lined by keratinizing squamous epithelium in the superficial dermis. The superficial lining epithelium and interfollicular epidermis were atrophic, while the deep epithelium showed mild proliferation and melanin pigmentation with a few short projections extending into the surrounding dermis. We diagnosed multiple Winer's dilated pores based on late-onset lesions and pathological features. This patient may represent the first case of multiple vulvar Winer's dilated pores. We suggest that electrocautery may be effective for treating this type of superficial entity. Keywords: Electrocoagulation; Neoplasms, adnexal and skin appendages; Pathology; Vulvar neoplasms
\end{abstract}

\section{INTRODUCTION}

Winer's dilated pore (WDP) is an uncommon appendageal tumor characterized by a dilated follicular pore filled with keratinous material. WDP usually appears as a giant comedone mainly involving the face, neck, and upper trunk in adults. Multiple lesions are occasionally reported..$^{1,2}$ On the other hand, nevus comedonicus (NC) is a rare developmental abnormality of the follicular infundibulum that mostly develops from birth to childhood. The grouped or linear comedonal papules are distributed commonly on the face, neck, trunk, and proximal limbs, and occasionally on the scalp, palm, and genitalia. ${ }^{2-4}$ Although WDP and NC represent distinct disorders, they have some overlapping clinicopathological features. ${ }^{5,6}$ We describe a postmenopausal woman with multiple vulvar lesions that clinically simulated an angiokeratoma but pathologically resembled WDP and NC.

\section{CASE REPORT}

We report a 57-year-old woman who presented with a 1-year history of several asymptomatic vulvar papules. Her past medical and family histories were unremarkable. Physical examination revealed 8 black, slightly elevated, keratotic, round papules distributed on both labia majora ( 5 on the left and 3 on the right). These papules lacked central plugs, ranging from $1-5 \mathrm{~mm}$ in diameter (Figure 1). The patient was successfully treated with electrocautery. We observed no recurrence in the 34-month follow-up.

An excisional biopsy revealed multiple cystic invaginations opening into the overlying epidermis in the superficial dermis (Figure 2). The cavities were filled with laminated keratinous material and a few hair fragments. The cysts were lined by squamous epithelium with a granular layer. The superficial lining epithelium and the interfollicular epidermis were atrophic, while the deep epithelium showed mild proliferation and melanin pigmentation with a few short projections extending into the surrounding dermis. No sebaceous gland lobules or vellus hair follicles were attached to the lining epithelium. Focal perifollicular and perivascular infiltrates of lymphohistiocytes were evident in the dermis (Figure 3).

\section{Received on 27.11.2015}

Approved by the Advisory Board and accepted for publication on 06.03.2016

* Work performed at the Department of Dermatology, Affiliated Hospital of Guangdong Medical University - Guangdong Province, China. Financial support: none.

Conflict of interest: none.

1 Department of Dermatology, Affiliated Hospital of Guangdong Medical University - Guangdong Province, China.

(C)2017 by Anais Brasileiros de Dermatologia 


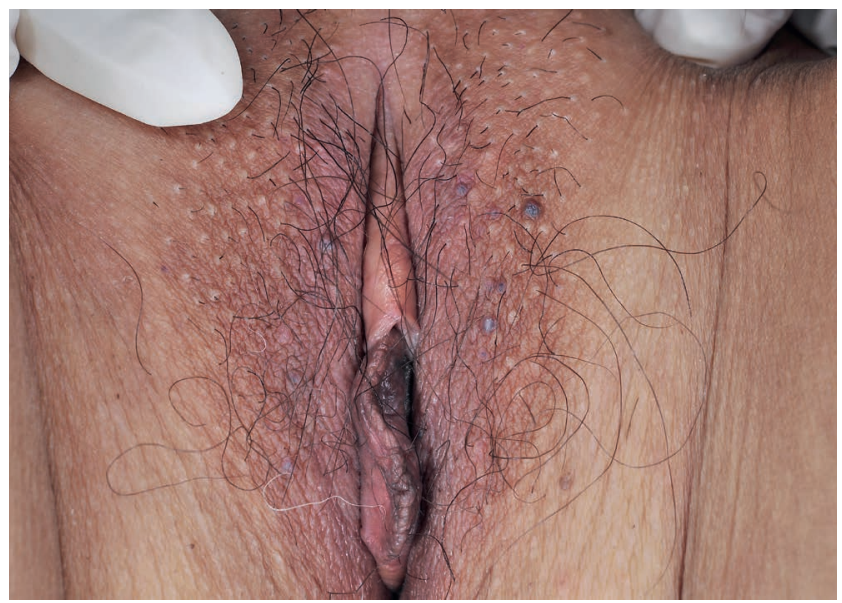

FIGURE 1: Several black round papules on both labia majora

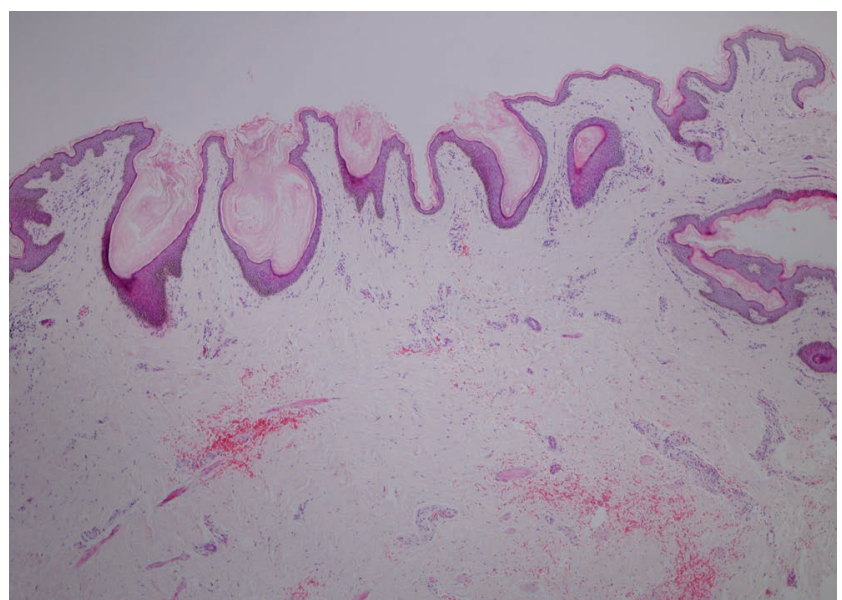

FIGURE 2: Multiple open dilated follicles filled with keratinous material in the superficial dermis (Hematoxylin \& eosin, X40)

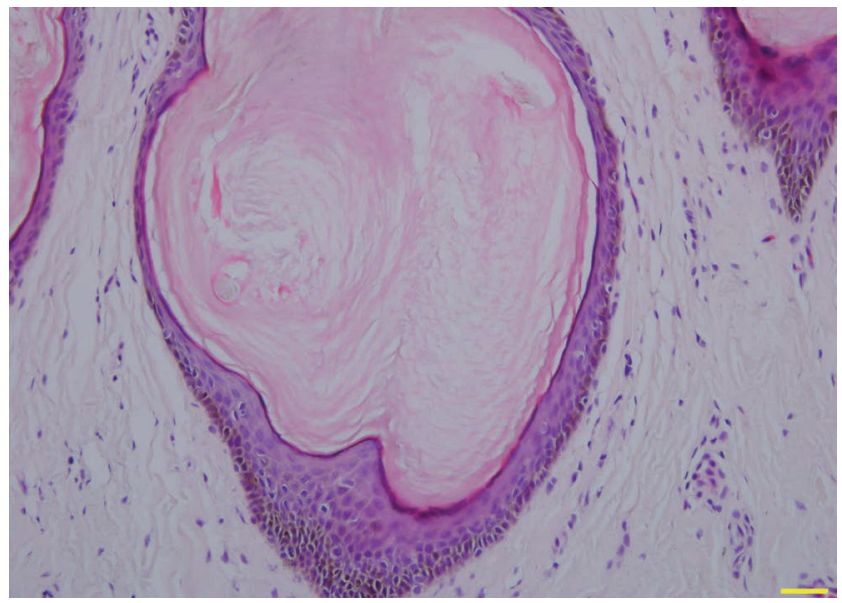

FIGURE 3: A dilated follicle filled with keratinous material and two hair fragments lined by atrophic and proliferative squamous epithelium (Hematoxylin \& eosin, X200)

\section{DISCUSSION}

WDP is a specific follicular infundibular cyst that differentiates toward the infundibulum both architecturally and cytologically. ${ }^{1}$ Although WDP is not frequently reported in the literature, we believe that most cases are not biopsied or reported due to the benign nature of this tumor. A comedonal papule or nodule is typical, but multiple lesions are seen in three cases. ${ }^{5-7}$ Each patient had one of the following concomitant diseases: squamous cell carcinoma, trichoblastoma, and trichoid basal cell carcinoma. ${ }^{8-10}$ Pathologically, a dilated infundibulum filled with keratinous material opens into the surface and extends into the superficial or deep dermis. The lining epithelium is atrophic superficially but proliferative deep in the cavity. The acanthotic epithelium, in turn, forms digitate projections into the surrounding dermis. Melanin pigmentation, small sebaceous lobules, and vellus hair follicles are sometimes observed. ${ }^{1,2}$ WDP can be divided into four types: funnel-shaped, balloon-shaped, multilobular with cystic compartments, and superficial with a widened infundibulum. ${ }^{1}$

NC is more common than WDP. About 200 NC cases had been reported up to 2007 since it was first described by Kofmann in $1895 .^{3}$ The comedonal lesions are mostly unilateral but rarely bilateral. NC can be divided into two types. The first type is non-pyogenic NC with comedo-like eruptions. The second type is NC with cysts, papules, pustules, and abscesses in various stages of development. NC can be associated with other cutaneous and internal defects (nevus comedonicus syndrome), and epithelial tumors., ${ }^{3,4}$ Pathologically, the grouped, dilated, keratin-filled invaginations are lined by keratinizing squamous epithelium. The epithelial lining is atrophic or mildly acanthotic. The interfollicular epidermis is frequently normal but may show hyperkeratosis and acanthosis. Some cases exhibit inflammation and dermal scarring. Rudimentary hair follicles and small sebaceous lobules may be present. ${ }^{2-5}$

Due to the several clinical and pathological similarities between WDP and NC, their differential diagnosis is not clear especially when multiple comedo-like lesions are present. Two patients with clinical presentations of NC and pathological features of WDP were diagnosed differently. ${ }^{5,6}$ Resnik et al. considered that the dilated pore nevus might be a histologic variant of NC because all of the published NC cases manifested multiple widely dilated keratin-filled follicular cysts without epithelial proliferation. ${ }^{5}$ Contrarily, Konohana \& Kobayashi named their case as aggregated dilated pores since WDP usually affected adults but not children. ${ }^{6}$

In our patient, asymptomatic black keratotic papules on both labia majora are firstly reminiscent of vulvar angiokeratoma, which can be excluded by pathological examination. Then, the pathological results of multiple dilated keratin-filled follicular invaginations lined with keratinizing squamous epithelium give rise to a differential diagnosis between WDP and NC. Resnik et al. proposed that the proliferative follicular epithelium may be peculiar to WDP but not to NC. ${ }^{5}$ The atrophy of superficial lining and mild proliferation of deep lining epithelium with fewer short projections and melanin pigmentation of the cystic wall are compatible with WDP, while multiple dilated follicular infundibula and insignificantly proliferative epithelium are akin to NC. We doubt that the slight proliferation of epithelial lining might be related to the short 
course of our case as compared with the long duration in patients with dilated pore nevus or aggregated dilated pores. ${ }^{5,6}$ In addition, WDP always appears in adulthood, while NC frequently develops in childhood. Considering all these facts, we thus accept the opinion of Konohana \& Kobayashi ${ }^{6}$ and believe that the diagnosis of multiple WDPs could be suitable for our case.

To our knowledge, this patient represents the first case of multiple vulvar WDPs. Although surgical excision is the first choice therapy for $\mathrm{WDP}^{7,8}$ electrocautery may also be effective for the superficial type of the entity.

\section{REFERENCES}

1. Steffen C. Winer's dilated pore: the infundibuloma. Am J Dermatopathol 2001;23:246-53.

2. Weedon D. Weedon's Skin Pathology. 3rd ed. New York: Churchill Livingston; 2010.

3. Guldbakke KK, Khachemoune A, Deng A, Sina B. Naevus comedonicus: a spectrum of body involvement. Clin Exp Dermatol. 2007;32:488-92.

4. Tchernev G, Ananiev J, Semkova K, Dourmishev LA, Schönlebe J, Wollina U. Nevus comedonicus: an updated review. Dermatol Ther (Heidelb). 2013;3:33-40.

5. Resnik KS, Kantor GR, Howe NR, Ditre CM. Dilated pore nevus. A histologic variant of nevus comedonicus. Am J Dermatopathol. 1993:15:169-71.

6. Konohana A, Kobayashi T. Aggregated dilated pores. J Dermatol. 1999;26:332-3.

7. Mittal RR, Sethi PS, Jha A. Dilated pore of Winer. Indian J Dermatol Venereol Leprol. 2002;68:239-40.

8. Zhao L, Xu J, Fang F, Qian G, Wang Y, Wang QQ. Squamous cell carcinoma found in a dilated pore. J Eur Acad Dermatol Venereol. 2007:21:277-8.

9. Misago N, Sada A, Narisawa Y. Trichoblastoma with a dilated pore. J Am Acad Dermatol. 2006;54:357-8.

10. Carlson-Sweet KL, Weigand DA, MacFarlane DF. Trichoid basal cell carcinoma found in a dilated pore on the nose. Dermatol Surg. 2000;26:874-6.

MAILING ADDRESS:

Yi-Ming Fan

Department of Dermatology,

Affiliated Hospital of Guangdong Medical University, Zhanjiang,

524001 Guangdong, China.

E-mail:ymfan1963@163.com

How to cite this article: Shi G, Li W, Liang N, Wang TT, Zhou Y, Fan YM. Multiple vulvar dilated pores in a postmenopausal woman. An Bras Dermtatol. 2017;92(4):562-4. 